\title{
LIGHTLIKE REAL HYPERSURFACES WITH TOTALLY UMBILICAL SCREEN DISTRIBUTIONS
}

\author{
DAE Ho JIN
}

\begin{abstract}
In this paper, we study the geometry of lightlike real hypersurfaces of an indefinite Kaehler manifold. The main result is a characterization theorem for lightlike real hypersurfaces $M$ of an indefinite complex space form $\bar{M}(c)$ such that the screen distribution is totally umbilic.
\end{abstract}

\section{Introduction}

It is well known that the normal bundle $T M^{\perp}$ of the lightlike hypersurface $M$ of a semi-Riemannian manifold $\bar{M}$ is a subbundle of the tangent bundle $T M$, of rank 1. Thus there exists a non-degenerate complementary vector bundle $S(T M)$ of $T M^{\perp}$ in $T M$, called a screen distribution on $M$, such that

$$
T M=T M^{\perp} \oplus_{\text {orth }} S(T M),
$$

where $\oplus_{\text {orth }}$ denotes the orthogonal direct sum. We denote such a lightlike hypersurface by $(M, g, S(T M))$. Denote by $F(M)$ the algebra of smooth functions on $M$ and by $\Gamma(E)$ the $F(M)$ module of smooth sections of a vector bundle $E$ over $M$. We use the same notation for any other vector bundle. We known [2] that, for any null section $\xi$ of $T M^{\perp}$ on a coordinate neighborhood $\mathcal{U} \subset M$, there exists a unique null section $N$ of a unique vector bundle $\operatorname{tr}(T M)$ in $S(T M)^{\perp}$ satisfying

$$
\bar{g}(\xi, N)=1, \bar{g}(N, N)=\bar{g}(N, X)=0, \forall X \in \Gamma(S(T M)) .
$$

Then the tangent bundle $T \bar{M}$ of $\bar{M}$ is decomposed as follow:

$$
T \bar{M}=T M \oplus \operatorname{tr}(T M)=\left\{T M^{\perp} \oplus \operatorname{tr}(T M)\right\} \oplus_{\text {orth }} S(T M) .
$$

We call $\operatorname{tr}(T M)$ and $N$ the transversal vector bundle and the null transversal vector field of $M$ with respect to $S(T M)$ respectively.

We recall that Tashiro-Tachibana [6] and Bejancu-Duggal [1] proved the nonexistence of totally umbilical non-degenerate and lightlike real hypersurfaces of an indefinite complex space form $\bar{M}(c)(c \neq 0)$ of constant holomorphic

Received June 18, 2009.

2000 Mathematics Subject Classification. Primary 53C25, 53C40, 53C50.

Key words and phrases. lightlike real hypersurface, totally umbilical distribution, indefinite complex space form. 
sectional curvature $c$, in case $\bar{g}$ is positive definite and indefinite respectively. In 1996, Duggal-Bejancu have proved the following theorem for lightlike real hypersurfaces of $\bar{M}(c)$ such that $S(T M)$ is totally umbilic in their book [2]:

Theorem A ([2]). Let $(M, g, S(T M))$ be a lightlike real hypersurface of $\bar{M}(c)$ such that $S(T M)$ is totally umbilic in $M$. Then $S(T M)$ is totally geodesic.

The purpose of this paper is to prove a new characterization theorem for lightlike real hypersurfaces $M$ of $\bar{M}(c)$ such that $S(T M)$ is totally umbilic:

Theorem 1.1. Let $(M, g, S(T M))$ be a lightlike real hypersurface of an indefinite complex space form $\bar{M}(c)$ such that $S(T M)$ is totally umbilic in $M$. Then we have both $c=0$ and $C=0$, on any $\mathcal{U} \subset M$. Moreover we show that

(1) $c=0$ implies that the ambient space $\bar{M}(c)$ is a semi-Euclidean space,

(2) $C=0$, on any $\mathcal{U} \subset M$, implies that $S(T M)$ is totally geodesic in $M$.

Comparing our Theorem 1.1 with above Theorem A, we observe that Theorem 1.1 has the following new features of geometric significance. We prove that the holomorphic sectional curvature $c$ satisfies $c=0$ if $S(T M)$ is totally umbilic in $M$. This is a very significant result. Contrary to this, there is no discussion on such a relationship in Theorem A's above result. Thus we also prove the non-existence of lightlike real hypersurfaces of $\bar{M}(c)(c \neq 0)$ such that $S(T M)$ is totally umbilic. For the rest of this paper, using Theorem 1.1, we prove several additional theorems for lightlike real hypersurfaces $M$ of $\bar{M}(c)$ such that $S(T M)$ is totally umbilic in $M$. Recall the following structure equations:

Let $\bar{\nabla}$ be the Levi-Civita connection of $\bar{M}$ and $P$ the projection morphism of $\Gamma(T M)$ on $\Gamma(S(T M))$ with respect to the decomposition (1.1). Then the local Gauss and Weingartan formulas are given by

$$
\begin{aligned}
& \bar{\nabla}_{X} Y=\nabla_{X} Y+B(X, Y) N, \\
& \bar{\nabla}_{X} N=-A_{N} X+\tau(X) N, \\
& \nabla_{X} P Y=\nabla_{X}^{*} P Y+C(X, P Y) \xi, \\
& \nabla_{X} \xi=-A_{\xi}^{*} X-\tau(X) \xi
\end{aligned}
$$

for any $X, Y \in \Gamma(T M)$, where $\nabla$ and $\nabla^{*}$ are the liner connections on $T M$ and $S(T M)$ respectively, $B$ and $C$ are the local second fundamental forms on $T M$ and $S(T M)$ respectively, $A_{N}$ and $A_{\xi}^{*}$ are the shape operators on $T M$ and $S(T M)$ respectively and $\tau$ is a 1 -form on $T M$.

Since $\bar{\nabla}$ is torsion-free, the induced connection $\nabla$ is also torsion-free and $B$ is symmetric. From the fact that $B(X, Y)=\bar{g}\left(\bar{\nabla}_{X} Y, \xi\right)$, we show that $B$ is independent of the choice of a screen distribution and satisfies

$$
B(X, \xi)=0, \quad \forall X \in \Gamma(T M) .
$$

The induced connection $\nabla$ of $M$ is not metric and satisfies

$$
\left(\nabla_{X} g\right)(Y, Z)=B(X, Y) \eta(Z)+B(X, Z) \eta(Y)
$$


for any $X, Y, Z \in \Gamma(T M)$, where $\eta$ is a 1-form on $T M$ such that

$$
\eta(X)=\bar{g}(X, N), \quad \forall X \in \Gamma(T M) .
$$

But the connection $\nabla^{*}$ on $S(T M)$ is metric. The above two local second fundamental forms $B$ and $C$ are related to their shape operators by

$$
\begin{array}{ll}
B(X, Y)=g\left(A_{\xi}^{*} X, Y\right), & \bar{g}\left(A_{\xi}^{*} X, N\right)=0, \\
C(X, P Y)=g\left(A_{N} X, P Y\right), & \bar{g}\left(A_{N} X, N\right)=0 .
\end{array}
$$

From (1.11), $A_{\xi}^{*}$ is $S(T M)$-valued and self-adjoint on $T M$ such that

$$
A_{\xi}^{*} \xi=0 .
$$

We denote by $\bar{R}, R$ and $R^{*}$ the curvature tensors of the Levi-Civita connection $\bar{\nabla}$ of $\bar{M}$, the induced connection $\nabla$ of $M$ and the connection $\nabla^{*}$ on $S(T M)$, respectively. Using the Gauss-Weingarten equations for $M$ and $S(T M)$, we obtain the Gauss-Codazzi equations for $M$ and $S(T M)$ such that, for any vector fields $X, Y, Z, W \in \Gamma(T M)$,

$$
\begin{aligned}
(1.14) \bar{g}(\bar{R}(X, Y) Z, P W)= & g(R(X, Y) Z, P W) \\
& +B(X, Z) C(Y, P W)-B(Y, Z) C(X, P W), \\
(1.15) \bar{g}(\bar{R}(X, Y) Z, \xi)= & g(R(X, Y) Z, \xi) \\
= & \left(\nabla_{X} B\right)(Y, Z)-\left(\nabla_{Y} B\right)(X, Z) \\
& +B(Y, Z) \tau(X)-B(X, Z) \tau(Y), \\
(1.16) \bar{g}(\bar{R}(X, Y) Z, N)=g( & R(X, Y) Z, N), \\
(1.17) g(R(X, Y) P Z, P W)= & g\left(R^{*}(X, Y) P Z, P W\right) \\
& +C(X, P Z) B(Y, P W)-C(Y, P Z) B(X, P W), \\
(1.18) g(R(X, Y) P Z, N)= & \left(\nabla_{X} C\right)(Y, P Z)-\left(\nabla_{Y} C\right)(X, P Z) \\
& +C(X, P Z) \tau(Y)-C(Y, P Z) \tau(X) .
\end{aligned}
$$

\section{Lightlike real hypersurfaces}

Let $\bar{M}=(\bar{M}, J, \bar{g})$ be a real $2 m$-dimensional indefinite Kaehler manifold, where $\bar{g}$ is a semi-Riemannian metric of index $q=2 v, 0<v<m$, and $J$ is an almost complex structure on $\bar{M}$ satisfying, for all $X, Y \in \Gamma(T \bar{M})$,

$$
J^{2}=-I, \quad \bar{g}(J X, J Y)=\bar{g}(X, Y), \quad\left(\bar{\nabla}_{X} J\right) Y=0 .
$$

An indefinite complex space form, denoted by $\bar{M}(c)$, is a connected indefinite Kaehler manifold of constant holomorphic sectional curvature $c$ such that

$$
\begin{gathered}
\bar{R}(X, Y) Z=\frac{c}{4}\{\bar{g}(Y, Z) X-\bar{g}(X, Z) Y+\bar{g}(J Y, Z) J X \\
-\bar{g}(J X, Z) J Y+2 \bar{g}(X, J Y) J Z\}
\end{gathered}
$$

for all $X, Y, Z \in \Gamma(T M)$. Let $(M, g, S(T M))$ be a lightlike real hypersurface of an indefinite Kaehler manifold $\bar{M}$, where $g$ is the degenerate induced metric of $M$. Then the screen distribution $S(T M)$ splits as follow [2]: 
Let $\{\xi, N\}$ be a pair of local sections of $T M^{\perp} \oplus \operatorname{tr}(T M)$. Then we have

$$
\bar{g}(J \xi, \xi)=\bar{g}(J \xi, N)=\bar{g}(J N, \xi)=\bar{g}(J N, N)=0, \bar{g}(J \xi, J N)=1 .
$$

This show that $J \xi$ and $J N$ are vector fields tangent to $M$. Thus $J\left(T M^{\perp}\right)$ and $J(\operatorname{tr}(T M))$ are distributions on $M$ of rank 1 such that $T M^{\perp} \cap J\left(T M^{\perp}\right)=$ $\{0\}$ and $T M^{\perp} \cap J(\operatorname{tr}(T M))=\{0\}$. Thus $J\left(T M^{\perp}\right) \oplus J(\operatorname{tr}(T M))$ is a vector subbundle of $S(T M)$ of rank 2 . There exists a non-degenerate almost complex distribution $D_{o}$ on $M$ with respect to $J$, i.e., $J\left(D_{o}\right)=D_{o}$, such that

$$
S(T M)=\left\{J\left(T M^{\perp}\right) \oplus J(\operatorname{tr}(T M))\right\} \oplus_{\text {orth }} D_{o} .
$$

Consider the 2-lightlike almost complex distribution $D$ such that

$$
D=\left\{T M^{\perp} \oplus_{\text {orth }} J\left(T M^{\perp}\right)\right\} \oplus_{\text {orth }} D_{o}, \quad T M=D \oplus J(\operatorname{tr}(T M))
$$

and the local lightlike vector fields $U$ and $V$ such that

$$
U=-J N, \quad V=-J \xi .
$$

Denote by $S$ the projection morphism of $T M$ on $D$. Then, by the second equation of (2.5)[(2.5)-2], any vector field on $M$ is expressed as follows

$$
X=S X+u(X) U, \quad J X=F X+u(X) N,
$$

where $u$ and $v$ are 1-forms locally defined on $M$ by

$$
u(X)=g(X, V), \quad v(X)=g(X, U)
$$

and $F$ is a tensor field of type $(1,1)$ globally defined on $M$ by

$$
F X=J S X, \forall X \in \Gamma(T M) .
$$

Differentiate (2.6)-1 with $X$ and use (1.5), (1.7), (2.1)-3 and (2.7)-2, we have

$$
\begin{aligned}
& B(X, U)=v\left(A_{\xi}^{*} X\right)=u\left(A_{N} X\right)=C(X, V), \forall X \in \Gamma(T M), \\
& \nabla_{X} U=F\left(A_{N} X\right)+\tau(X) U, \quad \nabla_{X} V=F\left(A_{\xi}^{*} X\right)-\tau(X) V .
\end{aligned}
$$

\section{Totally umbilical screen distributions}

Definition 1. We say that (each integral leaf of) $S(T M)$ is totally umbilic[2] in $M$ if, on any coordinate neighborhood $\mathcal{U} \subset M$, there is a smooth function $\gamma$ such that $A_{N} X=\gamma P X$ for any $X \in \Gamma(T M)$, or equivalently,

$$
C(X, P Y)=\gamma g(X, Y)
$$

for all $X, Y \in \Gamma(T M)$. In case $\gamma=0$ (or $\gamma \neq 0)$ on $\mathcal{U}$, we say that $S(T M)$ is totally geodesic (or proper totally umbilic) in $M$.

In general, $S(T M)$ is not necessarily integrable. The following result gives equivalent conditions for the integrability of $S(T M)$ : 
Theorem $3.1([2,3])$. Let $(M, g, S(T M))$ be a lightlike hypersurface of a semiRiemannian manifold $(\bar{M}, \bar{g})$. Then the following are equivalent:

(1) $S(T M)$ is integrable.

(2) $C$ is symmetric on $\Gamma(S(T M))$.

(3) $A_{N}$ is self-adjoint on $\Gamma(S(T M))$ with respect to $g$.

Note 1. If $S(T M)$ is totally umbilic in $M$, then $C$ is symmetric on $\Gamma(S(T M))$. Thus, by Theorem 3.1, $S(T M)$ is integrable and $M$ is locally a product manifold $L_{\xi} \times M^{*}$, where $L_{\xi}$ is a null curve and $M^{*}$ is a leaf of $S(T M)[2,3]$.

Proof of Theorem 1.1. Using the equations (2.9) and (3.1), we have

$$
B(X, U)=\gamma g(X, V), \quad \forall X \in \Gamma(T M) .
$$

Replace $X$ by $U$ and $V$ by turns in (3.2), we obtain

$$
B(U, U)=\gamma, \quad B(U, V)=0 .
$$

From (1.9), (1.16), (1.18) and (3.1), for any $X, Y, Z \in \Gamma(T M)$, we obtain

$$
\begin{aligned}
& \gamma B(Y, P Z) \eta(X)-\left\{X[\gamma]-\gamma \tau(X)-\frac{c}{4} \eta(X)\right\} g(Y, P Z) \\
= & \gamma B(X, P Z) \eta(Y)-\left\{Y[\gamma]-\gamma \tau(Y)-\frac{c}{4} \eta(Y)\right\} g(X, P Z) \\
& +\frac{c}{4}\{\bar{g}(J X, P Z) v(Y)-\bar{g}(J Y, P Z) v(X)-2 \bar{g}(X, J Y) v(P Z)\} .
\end{aligned}
$$

Replacing $X$ by $\xi$ in this equation and using (1.8), (2.6) and (2.8), we have

$$
\begin{aligned}
\gamma B(Y, P Z)= & \left\{\xi[\gamma]-\gamma \tau(\xi)-\frac{c}{4}\right\} g(Y, P Z) \\
& -\frac{c}{4}\{u(P Z) v(Y)+2 u(Y) v(P Z)\}, \forall Y, Z \in \Gamma(T M) .
\end{aligned}
$$

Taking $Y=U, P Z=V ; Y=V, P Z=U$ and $Y=P Z=U$ by turns in (3.4), and then, use (2.8) and (3.3), we have

$$
\xi[\gamma]-\gamma \tau(\xi)-\frac{3 c}{4}=0, \quad \xi[\gamma]-\gamma \tau(\xi)-\frac{c}{2}=0, \quad \gamma^{2}=0
$$

respectively. This shows that $c=0$ and $\gamma=0$. Thus we have Theorem 1.1.

Corollary 1. There exist no lightlike real hypersurfaces of an indefinite complex space form $\bar{M}(c)(c \neq 0)$ such that $S(T M)$ is totally umbilic in $M$.

Corollary 2. There exist no lightlike real hypersurfaces of an indefinite complex space form $\bar{M}(c)$ such that $S(T M)$ is proper totally umbilic.

Proposition 3.2. Let $(M, g, S(T M))$ be a lightlike real hypersurface of an indefinite complex space form $\bar{M}(c)$. If $S(T M)$ is totally umbilic in $M$, then the vector field $U$ is conjugate to any vector field on $M$. In particular, $U$ is an asymptotic vector field. Moreover, $B$ is degenerate on $\Gamma(S(T M))$.

Proof. Since $\gamma=0$ on any $\mathcal{U} \subset M$, from (3.2), we have $B(X, U)=0$ for any $X \in \Gamma(T M)$. Thus we have our assertion. 
Theorem 3.3. Let $(M, g, S(T M))$ be a lightlike real hypersurface of an indefinite complex space form $\bar{M}(c)$ such that $S(T M)$ is totally umbilic in $M$. Then $H=D_{o} \oplus_{\text {orth }} J(\operatorname{tr}(T M)) \oplus_{\text {orth }} T M^{\perp}$ is a parallel distribution with respect to the induced connection $\nabla$ and $M$ is locally a product manifold $L_{v} \times M^{\natural}$, where $L_{v}$ is a null curve tangent to $J\left(T M^{\perp}\right)$ and $M^{\natural}$ is a leaf of $H$.

Proof. In general, by using (1.4), (2.1) and (2.10), we derive

$$
\begin{aligned}
& g\left(\nabla_{X} \xi, U\right)=-g\left(\xi, \bar{\nabla}_{X} U\right)=-B(X, U), \quad g\left(\nabla_{X} U, U\right)=0, \\
& g\left(\nabla_{X} Y, U\right)=-g\left(Y, \bar{\nabla}_{X} U\right)=-g\left(Y, \nabla_{X} U\right)=0
\end{aligned}
$$

for all $X \in \Gamma(T M)$ and $Y \in \Gamma\left(D_{o}\right)$. Since $S(T M)$ is totally umbilic in $M$, we have $B(X, U)=0$ by $(3.2)$ with $\gamma=0$. Thus $H$ is parallel with respect to $\nabla$ and both $H$ and $J\left(T M^{\perp}\right)$ are integrable distributions. Thus we obtain our theorem.

Definition 2. We say that $M$ is totally umbilic $[2]$ in $\bar{M}$ if, on any coordinate neighborhood $\mathcal{U} \subset M$, there is a smooth function $\beta$ such that

$$
B(X, Y)=\beta g(X, Y)
$$

for all $X, Y \in \Gamma(T M)$. In case $\beta=0$ on $\mathcal{U}$, we say that $M$ is totally geodesic.

Theorem 3.4. Let $(M, g, S(T M))$ be a totally umbilical lightlike real hypersurface of an indefinite complex space form $\bar{M}(c)$ such that $S(T M)$ is totally umbilic in $M$. Then $M$ is totally geodesic in $\bar{M}$.

Proof. From the equations (3.2) with $\gamma=0$ and (3.5), we show that

$$
\beta g(X, U)=B(X, U)=0
$$

for all $X \in \Gamma(T M)$. Replacing $X$ by $V$ in (3.6), we have $\beta=0$, i.e., $B=0$. Thus $M$ is totally geodesic in $\bar{M}$. Therefore, we have our theorem.

Theorem 3.5. Let $(M, g, S(T M))$ be a totally umbilical lightlike real hypersurface of an indefinite complex space form $\bar{M}(c)$ such that $S(T M)$ is totally umbilic in $M$. Then $D$ is a parallel distribution with respect to the induced connection $\nabla$ and $M$ is locally a product manifold $L_{u} \times M^{\sharp}$, where $L_{u}$ is a null curve tangent to $J(\operatorname{tr}(T M))$ and $M^{\sharp}$ is a leaf of $D$.

Proof. In general, by using (1.4), (2.1) and (2.10), we derive

$$
\begin{aligned}
& g\left(\nabla_{X} \xi, V\right)=-g\left(\xi, \bar{\nabla}_{X} V\right)=-B(X, V), \quad g\left(\nabla_{X} V, V\right)=0, \\
& g\left(\nabla_{X} Y, V\right)=-g\left(Y, \nabla_{X} V\right)=-g\left(Y, F\left(A_{\xi}^{*} X\right)\right)=B(X, F Y)
\end{aligned}
$$

for all $X \in \Gamma(T M)$ and $Y \in \Gamma\left(D_{o}\right)$. Since $M$ is totally umbilic, we have $B=0$ by Theorem 3.4. Thus $D$ is parallel with respect to $\nabla$ and both $D$ and $J(\operatorname{tr}(T M))$ are integrable distributions. Thus we obtain our theorem.

Theorem 3.6. Let $(M, g, S(T M))$ be a lightlike real hypersurface of an indefinite complex space form $\bar{M}(c)$ such that $S(T M)$ is totally umbilic in $M$. Then $M$ and each leaf $M^{*}$ of $S(T M)$ are spaces of constant curvature 0. 
Proof. Consider the induced quasi-orthonormal frame field $\left\{\xi ; W_{a}\right\}$ on $M$ such that $\operatorname{Rad}(T M)=\operatorname{Span}\{\xi\}$ and $S(T M)=\operatorname{Span}\left\{W_{a}\right\}$. Using this quasi-orthonormal frame field, we obtain

$$
R(X, Y) Z=\sum_{a=1}^{2 m-2} \epsilon_{a} g\left(R(X, Y) Z, W_{a}\right) W_{a}+g(R(X, Y) Z, N) \xi
$$

for any $X, Y \in \Gamma(T M)$ and $\epsilon_{a}=g\left(W_{a}, W_{a}\right)$. Using (1.14), (1.16) and the last equation, we have $R(X, Y) Z=0$ for any $X, Y, Z \in \Gamma(T M)$, due to the facts that $c=0$ and $C=0$ by Theorem 1.1. Thus $M$ is a space of constant curvature 0. Also, from (1.14) and (1.17), we also have $R^{*}(X, Y) Z=0$ for any $X, Y, Z \in \Gamma(S(T M))$. Thus $M^{*}$ is also a space of constant curvature 0 .

Combining Note 1 and Theorem 1.1 and 3.6, we have:

Theorem 3.7. Let $(M, g, S(T M))$ be a lightlike real hypersurface of an indefinite complex space form $\bar{M}(c)$ such that $S(T M)$ is totally umbilic in $M$. Then $M$ is a lightlike space form of constant curvature 0 and locally a product manifold $L_{\xi} \times M^{*}$, where $L_{\xi}$ is a null curve and $M^{*}$ is a semi-Euclidean space.

Nomizu and Pinkall [4] defined an affine immersion as follows: Let $f: M \rightarrow$ $\bar{M}$ be an immersion of a manifold $M$ as a hypersurface of a manifold $\bar{M}$ and $\nabla$ and $\bar{\nabla}$ be torsion-free linear connections on $M$ and $\bar{M}$ respectively. Then $f$ is an affine immersion if there exists locally a transversal vector field $N$ along $f$ such that

$$
\bar{\nabla}_{f_{*} X} f_{*} Y=f_{*}\left(\nabla_{X} Y\right)+B(X, Y) N, \quad \forall X, Y \in \Gamma(T M),
$$

where $f_{*}$ is the differential map of $f$. Then, as usual, we put

$$
\bar{\nabla}_{f_{*} X} N=-A_{N}\left(f_{*} X\right)+\tau\left(f_{*} X\right) N .
$$

Clearly, by (1.4), any lightlike isometric immersion is an affine immersion. Suppose $\operatorname{dim} M=2 m-1$ and $\nabla$ is a flat connection on $M$. Let $\psi: M \rightarrow \mathbb{R}^{2 m-1}$ such that every point $x \in M$ has a neighborhood $\mathcal{U}$ on which $\psi$ is an affine connection preserving differmorphism with an open neighborhood $\mathcal{W}$ of $\psi(x)$ in $\mathbb{R}^{2 m-1}$. Consider $\mathbb{R}^{2 m-1}$ as a hyperplane of $\mathbb{R}^{2 m}$ and let $N$ be a parallel vector field transversal to $\mathbb{R}^{2 m-1}$. Define, for any differentiable function $F: M \rightarrow \mathbb{R}$,

$$
f: M \rightarrow \mathbb{R}^{2 m} ; f(x)=\psi(x)+F(x) N, \quad \forall x \in M .
$$

Thus, $f$ is an affine immersion with $A_{N}=0$, called the graph immersion with respect to $F$. Now, we recall the following result.

Theorem $3.8([2])$. Let $M$ be a lightlike hypersurface of $\mathbb{R}_{q}^{2 m}$ with a parallel screen distribution $S(T M)$. Then the immersion of $M$ is affinely equivalent to the graph immersion of a certain function $F: M \rightarrow \mathbb{R}$.

Theorem 3.9. Let $(M, g, S(T M))$ be a lightlike real hypersurface of an indefinite complex space form $\bar{M}(c)$ such that $S(T M)$ is totally umbilic in $M$. Then 
the immersion of $M$ is affinely equivalent to the graph immersion of a certain function $F: M \rightarrow \mathbb{R}$.

Proof. By Theorem 1.1, we have $C=0$, on any $\mathcal{U} \subset M$, and $c=0$, i.e., the screen distribution $S(T M)$ is totally geodesic in $M$ and the constant holomorphic sectional curvature $c$ of the ambient space $\bar{M}(c)$ satisfies $c=0$. By (1.6), $C=0$, on any $\mathcal{U} \subset M$, implies $S(T M)$ is parallel with respect to the induced connection $\nabla$. Also $c=0$ implies that the ambient space $\bar{M}(c)$ is $\mathbb{R}_{q}^{2 m}$. Therefore, by Theorem 3.8, we have our theorem.

\section{References}

[1] A. Bejancu and K. L. Duggal, Real hypersurfaces of indefinite Kaehler manifolds, Internat. J. Math. Math. Sci. 16 (1993), no. 3, 545-556.

[2] K. L. Duggal and A. Bejancu,Lightlike Submanifolds of Semi-Riemannian Manifolds and Applications, Mathematics and its Applications, 364. Kluwer Academic Publishers Group, Dordrecht, 1996.

[3] K. L. Duggal and D. H. Jin, Null curves and Hypersurfaces of Semi-Riemannian Manifolds, World Scientific Publishing Co. Pte. Ltd., Hackensack, NJ, 2007.

[4] K. Nomizu and U. Pinkall, On the geometry of affine immersions, Math. Z. 195 (1987), no. $2,165-178$

[5] B. O'Neill, Semi-Riemannian Geometry with Applications to Relativity, Academic Press, 1983

[6] Y. Tashiro and S. I. Tachibana, On Fubinian and C-Fubinian manifolds, Kōdai Math. Sem. Rep. 15 (1963), 176-183.

Department of Mathematics

DONGGUK UNIVERSITY

KYONGJU 780-714, KoreA

E-mail address: jindh@dongguk.ac.kr 